\title{
Sfruttamento idropotabile e geotermico: una convivenza possibile?
}

\author{
Nicola Cempini \\ Ingegnerie Toscane srl. \\ n.cempini@ingegnerietoscane.net
}

Oggi giustamente c'è un sempre maggior interesse verso la geotermia; lo sfruttamento dell'acqua sotterranea sia per riscaldare che per raffreddare è, grazie all'evoluzione delle tecnologie, una possibilità concreta una grande occasione per diminuire la dipendenza del nostro Paese dall'importazione di combustibili fossili quali petrolio e gas.

Come è noto si può sfruttare la cosiddetta bassa entalpia sia attraverso sonde geotermiche, senza portare in superficie l'acqua sotterranea, o con impianti di estrazione e re-iniezione (detti open loop) che al contrario captano il fluido con pozzi di estrazione, lo trasferiscono in superficie per il necessario scambio energetico e quindi lo riconvogliano nell'acquifero coi pozzi di re-immissione. Tutti noi ci auguriamo che lo sfruttamento della bassa entalpia, appena iniziato, continui e si sviluppi sempre di più; se questo avverrà le perforazioni dei primi 200-300 m aumenteranno molto. Si tratta di una occasione di crescita importante sia per il Paese, per i motivi energetici ricordati sopra, sia per il settore delle perforazioni ma che deve essere affrontata con grande rigore professionale.

Non mi stancherò mai di ripetere che la perforazione di un pozzo rappresenta una perturbazione importante dell'ambiente naturale ed è quindi di fondamentale importanza che le caratteristiche di protezione delle falde captate siano mantenute innanzitutto attraverso la corretta esecuzione di tamponi e cementazioni e più in generale con una esecuzione impeccabile che significa avere anche una progettazione e direzione lavori di livello adeguato.

Nel nostro Paese la maggior parte dell'acqua distribuita dagli acquedotti civili ma anche l'acqua utilizzata dall'industria e dall'agricoltura ha origine sotterranea; questi stessi acquiferi nella maggior parte dei casi sono gli stessi che possono essere sfruttati a fini geotermici (bassa entalpia). Bisogna allora porsi una domanda: è possibile il contestuale sfruttamento della stessa risorsa a scopo idropotabile e geotermico? Io penso di si, a patto che si rispettino determinate condizioni.

Prima di tutto è necessaria una conoscenza approfondita del territorio; la geometria degli acquiferi; la chimica delle acque; i parametri idrodinamici; la piezometria: sono questi i dati che servono per capire come si muove l'acqua sotterranea e costruire un modello concettuale che sarà alla base della modellazione, il secondo passaggio fondamentale.

Con la modellazione matematica infatti si è in grado di progettare correttamente i pompaggi sia in termini di posizione che di quantità, prevedere le interferenze e l'evoluzione del sistema acquifero nel tempo da tutti i punti di vista compresa la temperatura. Oggi infatti esistono software che consentono, ad esempio nel caso di un impianto open loop, di prevedere l'effetto che avrà lo sfruttamento geotermico sulla temperatura dell'acquifero nel tempo.

La terza condizione fondamentale è il monitoraggio; bisogna progettare e realizzare sistemi di monitoraggio delle acque sotterranee che confermino (o meno) nel tempo la correttezza delle previsioni progettuali e che consentano l'eventuale raffinazione del sistema. Un efficiente sistema di monitoraggio si deve basare su sonde piezometriche opportunamente piazzate e pluviometri. Sono dell'avviso che un sistema corretto di monitoraggio della falda non può prescindere dall'aspetto idrochimico e quindi è necessario procedere con cadenza temporale sufficiente al campionamento ed analisi di una serie adeguata di punti fissi per verificare l'andamento delle caratteristiche chimiche dell'acqua nel tempo. Ovviamente l'acqua sotterranea non cambia facies ma il pompaggio può determinare la mobilizzazione di sezioni dell'acquifero con caratteristiche di Eh, pH, temperatura, conducibilità e concentrazione di ioni Fe e Mn ad esempio diverse.

La quarta condizione, di cui ho già iniziato a parlare in apertura di questo articolo, è quella di eseguire a regola d'arte pozzi e perforazioni.

Oggi, date le condizioni economiche generali del nostro Paese, c'è una forte spinta da parte delle imprese ma anche delle stazioni appaltanti a contrarre i costi. Questo mal si concilia con una attività, la perforazione, il cui primo target deve essere quello della protezione ambientale. Tra l'altro la maggior parte delle imprese esecutrici e praticamente tutte le stazioni appaltanti possiedono la certificazione ISO 9001 e ISO 14001 e questo stona col bando di gare al massimo ribasso; è vero che la stazione appaltante determina la soglia di ribasso oltre la quale esclude le offerte anomale ma i criteri di ricerca dell'impresa esecutrice devono essere più ampi e comprendere aspetti quali l'investimento nella formazione professionale del personale (numero di patentini); il parco macchine a disposizione e soprattutto il curriculum.

Fermo restando che la realizzazione di qualsiasi tipo di pozzo deve garantire un elevato livello di protezione ambientale, occorre fare un distinguo tra quello che è un semplice pozzo domestico, definibile "da giardino", e un pozzo industriale, agricolo o idropotabile. Nel primo caso abbiamo a che fare con opere semplici, destinate a lavorare qualche decina di ore all'anno la cui acqua non concorre alla formazione del reddito dell'utilizzatore (uno dei criteri che la giurisprudenza detta per l'attribuzione della caratteristica di pozzo domestico). Nel secondo caso si tratta di opere che devono produrre una congrua quantità di acqua col minor dispendio energetico possibile e che devono garantire continuità a un processo indu- 
striale, a un piano di coltivazione o all'acquedotto civile. Tali pozzi dunque sono progettati, realizzati e testati per lavorare 12/18 ore al giorno tutti i giorni dell'anno. Va da sé che il costo di questo tipo di opere è molto superiore e che le imprese esecutrici devono avere un grado adeguato di specializzazione e esperienza.

In Italia le imprese esecutrici di pozzi per acqua devono iscriversi alla categoria SOA OS21 - opere strutturali speciali - che tuttavia comprende un grande numero di imprese esercenti le più disparate lavorazioni; fondazioni, palificazioni, muri di sostegno, ancoraggi, messa in sicurezza di pendii e frane, drenaggi e altro ancora. E' chiaro che il solo requisito dell'appartenenza alla OS21 non può essere di per se stesso sufficiente a garantire la selezione di una impresa professionalmente adeguata anche perché proprio in virtù della crisi scatta un meccanismo per cui qualcuno partecipa alla gara pur non avendo la struttura tecnica per la lavorazione specifi- ca di esecuzione di pozzi per acqua nel tentativo di prendere comunque un lavoro.

Stazioni appaltanti pubbliche e private devono individuare un compromesso soddisfacente tra le capacità tecniche dei contractors e un risultato economico congruo. Questo risultato si ottiene prima di tutto con progetti fatti bene e quindi con capitolati tecnici e bandi di gare che ben lungi da porre condizioni vessatorie devono essere confezionati in modo da individuare le imprese più qualificate.

Lacqua sotterranea fa parte di un ambiente naturale estremamente delicato; ristabilirne l'equilibrio dopo un inquinamento richiede anni e investimenti importanti. Se vogliamo continuare a sfruttare gli acquiferi a scopo idropotabile e industriale e addirittura implementarne lo sfruttamento dal punto di vista geotermico dobbiamo poter contare su tecnici e imprese di elevata preparazione professionale ma l'iniziativa non può essere lasciata alla buona volontà dei singoli; il quadro normativo deve evolvere in questo senso. 